\title{
Actividad Antimicrobiana Y Antioxidante de Hierbas Aromáticas Aplicables al Proceso de Marinado de Productos Cárnicos
}

Ricardo Sobol (I), Verónica Berges Soubies (I), Natalia Camardella (I), Fernando Reina (II), Graciela De Seta (II), Patricia Della Rocca (II), Silvia Raffellini (III,I), María Angélica Bianchi (II)

(I) UADE - Universidad Argentina de la Empresa (Lima 775 - Buenos Aires - Argentina), (II) UTN - Universidad Tecnológica Nacional (Medrano 951 - Buenos Aires - Argentina), (III) UNLu - Universidad Nacional de Luján (Ruta 5 y 7 - Luján - Buenos Aires - Argentina)

\section{Resumo}

Una de las prácticas empleadas en productos cárnicos es el marinado, que consiste en la maceración en una solución que contiene aditivos, saborizantes e ingredientes naturales, durante un período de tiempo, y que tiene como objetivo mejorar su sabor y conservación. Entre los ingredientes que se pueden aplicar se destacan las hierbas aromáticas que contribuirán a las características de palatabilidad del producto, y además podrían funcionar como barrera adicional para la inhibición de microorganismos patógenos, así como para limitar la oxidación de lípidos, una de las causas de deterioro químico en carnes. El objetivo de este trabajo fue estudiar la actividad antimicrobiana y antioxidante de extractos etanólicos de plantas aromáticas que podrían emplearse en procesos de marinado de carnes. Las hierbas aromáticas ensayadas fueron: orégano (Origanum vulgare), romero (Rosmarinus officinalis), salvia (Salvia officinalis) y tomillo (Thymus vulgaris). Los extractos se prepararon en una concentración de $50 \% \mathrm{~m} / \mathrm{m}$ empleando etanol absoluto como solvente de extracción. Se evaluó la actividad antimicrobiana de los extractos contra Listeria monocytogenes ATCC 7644 y Salmonella Abony NCTC 6017, utilizando el método de difusión en agar. En los extractos con actividad antimicrobiana, se evaluó la capacidad antioxidante mediante la cuantificación de polifenoles totales

\footnotetext{
Referência:

Ricardo Sobol, Verónica Berges Soubies, Natalia Camardella, Fernando Reina, Graciela De Seta, Patricia Della Rocca, Silvia Raffellini, María Angélica Bianchi. Actividad Antimicrobiana Y Antioxidante de Hierbas Aromáticas Aplicables Al Proceso de Marinado de Productos Cárnicos. In: Anais do 12 Congresso Latinoamericano de Microbiologia e Higiene de Alimentos - MICROAL 2014 [= Blucher Food Science Proceedings, num.1, vol.1]. São Paulo: Editora Blucher, 2014. 
por espectrofotometría (método Folin-Ciocalteau), y la identificación de grupos de componentes con características antioxidantes por espectrofotometría IR por Transformada de Fourier (FTIR). Los extractos no presentaron actividad antimicrobiana contra Salmonella. Sin embargo, los extractos de romero, salvia y tomillo presentaron actividad inhibitoria contra Listeria monocytogenes, siendo el primero el que presentó mayor capacidad antimicrobiana. El contenido de polifenoles de los extractos de romero, tomillo y salvia fue de $3.174,4$ ppm, 854,3 ppm y 348,9 ppm, respectivamente. El análisis por FTIR aportó perfiles similares para los tres extractos, con bandas indicativas de presencia de sustancias polifenólicas. Además, en los extractos de tomillo y salvia también aparecieron bandas indicativas de presencia de anillos lactona, flavonoides y/o cumarinas. La mayor actividad antimicrobiana y antioxidante detectada en el extracto de romero alienta su utilización en el marinado de carnes.

Palavras-Chave: Antimicrobianos naturales, Hierbas aromáticas, Productos cárnicos

Agência de Fomento: 